\title{
Forward-backward asymmetry of top quark in diquark models
}

\author{
Abdesslam Arhrib ${ }^{1,2 *}$, Rachid Benbrik ${ }^{2,3,4 \dagger}$ and Chuan-Hung Chen ${ }^{3,4 \ddagger}$ \\ 1 Dépatement de Mathématique, Faculté des Sciences and Techniques, \\ Université Abdelmalek Essâ̂di, B. 416, Tangier, Morocco \\ 2 LPHEA, Faculté des Sciences-Semlalia, B.P. 2390 Marrakesh, Morocco \\ 3 Department of Physics, National Cheng-Kung University, Tainan 701, Taiwan \\ ${ }^{4}$ National Center for Theoretical Sciences, Hsinchu 300, Taiwan
}

(Dated: May 30, 2018)

\begin{abstract}
Motivated by the recent unexpected large forward-backward asymmetry (FBA) of the top quark observed by $\mathrm{D} \varnothing$ and $\mathrm{CDF}$ at the Tevatron, we investigate a possible explanation for the anomaly within the framework of diquark models. In the diquark models, the top-quark pair production is mediated by the u-channel diagram. It is found that the color-triplet diquark can generate the FBA of $20 \%$ when the constraint from the cross section of the top-quark pair production is taken into account.

PACS numbers: 12.39.-x, 14.65.Ha
\end{abstract}

\footnotetext{
* Email:aarhrib@ictp.it

† Email:rbenbrik@mail.ncku.edu.tw

‡ Email:physchen@mail.ncku.edu.tw
} 
Although most experimental data are consistent with the Standard Model (SM) predictions, it is believed that the SM is just an effective theory of a more fundamental one yet to be discovered. For a correct understanding of the hierarchy problem of the Higgs mass and Planck scale, neutrinos masses, matter-antimatter asymmetry, dark matter, etc, new physics beyond the SM should be included. Theorists and experimentalists have put a lot of effort to investigate the existence of new physics. If such new physics exists, it can be probed either directly at collider or indirectly through precise measurements. Intriguingly, the new measurements at Tevatron on the forward-backward asymmetry (FBA) in the top-quark pair production at $\sqrt{s}=1.96 \mathrm{TeV}$ may provide the clue for the existence of new physics.

When D Collaboration published the first measurement on the FBA in top-quark pair production in the $p \bar{p}$ laboratory frame with $0.9 \mathrm{fb}^{-1}$ of data, an unexpected large FBA of top-quark was found [1]. Subsequently, CDF also observed the same phenomenon by using $1.9 \mathrm{fb}^{-1}[2]$. Furthermore, with an integrated luminosity of $3.2 \mathrm{fb}^{-1}$, the updated CDF's result in the $p \bar{p}$ laboratory frame is given by [3]

$$
A_{F B}^{t}=0.193 \pm 0.065 \text { (stat) } \pm 0.024 \text { (syst) }
$$

The indication of large FBA of top-quark is not smeared by statistic. In the SM, since the top-quark pair production is dominated by the strong interaction QCD contribution, due to C-parity invariance, a vanishing FBA at the leading order (LO) is expected. However, a nonvanishing FBA can be induced at the next-to-leading order (NLO) [4] and is given by $A_{\mathrm{FB}}^{t}=0.05 \pm 0.015$. Comparing the SM prediction with the latest CDF's measurement, although the deviation of data from the SM result is less than $3 \sigma$, the difference could actually originate from new physics contributions. Motivated by the recent measurements of $\mathrm{D} \varnothing$ and CDF at Tevatron, several possible explanations to the observed FBA have been proposed in several studies. [5-11]]. In this paper, we investigate the impact of colored scalars "diquarks" on the FBA of the $t \bar{t}$ production at Tevatron, where the diquark will contribute to the $t \bar{t}$ production through a $\mathrm{u}$-channel diagram.

It is known that the scalar sector of the SM has not been tested directly by any experiments, it is plausible to assume the existence of other possible scalars within $S U(3)_{c} \times S U(2)_{L} \times U(1)_{Y}$ gauge symmetry. Theoretically, exotic color states are well motivated. For example, one can find: color non-singlet scalar fields for CP violation [12, 13], color scalar quarks in supersymmetry with R-parity violation [14], supersymmet- 
ric $S U(2)_{L} \times S U(2)_{R} \times S U(4)_{c}$ model that embeds the seesaw mechanism for neutrino masses where a color sextet Higgs fields appears at TeV range [15]. Consequently, the general scalar representations could be $(\mathbf{1}, \mathbf{2})_{1 / 2},(\mathbf{8}, \mathbf{2})_{1 / 2},(\mathbf{6}, \mathbf{3})_{1 / 3},(\mathbf{6}, \mathbf{1})_{4 / 3,1 / 3,-2 / 3},(\mathbf{3}, \mathbf{3})_{-1 / 3}$, $(\mathbf{3}, \mathbf{1})_{2 / 3,-1 / 3,-4 / 3}$, where the first (second) argument in the brackets denotes the representation in color (weak isospin) space and the number in the subscript corresponds to the hypercharge of $U(1)_{Y}[12,13,16]$. Besides the SM Higgs doublet, it has been shown in Ref. [16] that when the hypothesis of minimal flavor violation (MFV) is imposed and if the scalar is flavor singlet [17], only the representation $(\mathbf{8}, \mathbf{2})_{1 / 2}$ could avoid FCNCs at the tree level. As a result, the couplings of color octet to quarks are associated with the quark masses. Therefore, the s-channel induced process $u \bar{u}(d \bar{d}) \rightarrow t \bar{t}$ in the color octet model is negligible. In addition, due to the suppression of Cabibbo-Kobayashi-Maskawa (CKM) matrix element $V_{t d}$, the t-channel $d \bar{d} \rightarrow t \bar{t}$ process induced by charged color-octet turns out to be rather small. In the following analysis, we will concentrate on the situation of color triplet and sextet.

For understanding what kind of colored scalars could contribute to FBA of top quark, we display various possible scalar diquarks in Table 1 [19], where the second column in the table denotes the representations of the diquarks under $S U(3)_{c} \times S U(2)_{L} \times U(1)_{Y}$, the third column gives the interactions of quarks and diquarks and the fourth column displays the relation of couplings in flavors. The notations used in the Table are as follows: the indices $(i, j)$ and $(\alpha, \beta)$ stand for the flavors and colors, $Q$ and $u(d)$ denote the $S U(2)_{L}$ doublet and singlet quarks, $\epsilon^{\alpha \beta \gamma} H_{\gamma}$ and $H^{\alpha \beta}$ represent the color triplet and sextet, respectively, $f^{c}=C \bar{f}^{T}$ with $C$ being the charge conjugation operator and $\epsilon=i \tau_{2}$. The involved couplings are considered as free parameters. From the table, we find that the $t \bar{t}$ pair in $p \bar{p}$ collisions can be produced via $d \bar{d} \rightarrow t \bar{t}$ and $u \bar{u} \rightarrow t \bar{t}$ processes, where the former is represented by models (1)-(5) while the latter is dictated by models (6) and (7). To be more specific, we sketch the associated Feynman diagrams in Fig. 1. Owing to the diquarks being color triplet and sextet in each diagram, for not showing the specific diquark, we have suppressed their color indices. In addition, one can also easily find that the couplings of diquarks to quarks are chiral, in other words, only left- or right-handed quarks are involved in the diquark models. Due to the chiral interactions, it is expected that a large FBA in $t \bar{t}$ production could be induced by the diquark contribution.

Since our purpose is to illustrate the impact of diquarks on the FBA of top quark produc- 
TABLE I: Various diquark models for the $d \bar{d} \rightarrow t \bar{t}$ and $u \bar{u} \rightarrow t \bar{t}$.

\begin{tabular}{cccc}
\hline \hline Model & $\mathrm{H}$ & Interaction & flavor symmetry \\
\hline$(1)$ & $(3,1,-1 / 3)$ & $f_{i j} \bar{Q}_{i \alpha}^{c} \epsilon Q_{j \beta} \varepsilon^{\alpha \beta \gamma} H_{\gamma}$ & $f_{i j}=f_{j i}$ \\
$(2)$ & $(3,1,-1 / 3)$ & $f_{i j} \bar{d}_{i \alpha} u_{j \beta}^{c} \varepsilon^{\alpha \beta \gamma} H_{\gamma}^{\dagger}$ & -- \\
$(3)$ & $f_{i j} \bar{Q}_{i \alpha}^{c} \epsilon Q_{j \beta} H^{\dagger \alpha \beta}$ & $f_{i j}=-f_{j i}$ \\
$(4)$ & $f_{i j} \bar{d}_{i \alpha} u_{j \beta}^{c} H^{\alpha \beta}$ & -- \\
$(5)$ & $(6,1,1 / 3)$ & $f_{i j} \bar{Q}_{i \alpha}^{c} \epsilon H_{\gamma} Q_{j \beta} \varepsilon^{\alpha \beta \gamma}$ & $f_{i j}=-f_{j i}$ \\
$(6)$ & $f_{i j} \bar{u}_{i \alpha} u_{j \beta}^{c} \varepsilon^{\alpha \beta \gamma} H_{\gamma}^{\dagger}$ & $f_{i j}=-f_{j i}$ \\
$(7)$ & $(3,3,-1 / 3)$ & $f_{i j} \bar{u}_{i \alpha} u_{j \beta}^{c} H^{\alpha \beta}$ & $f_{i j}=f_{j i}$ \\
\hline \hline
\end{tabular}

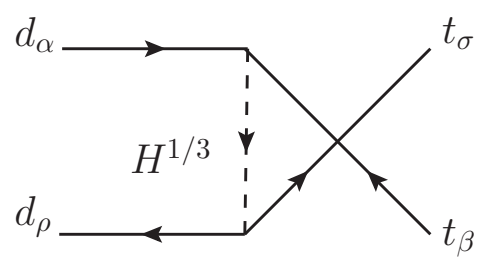

$(a)$

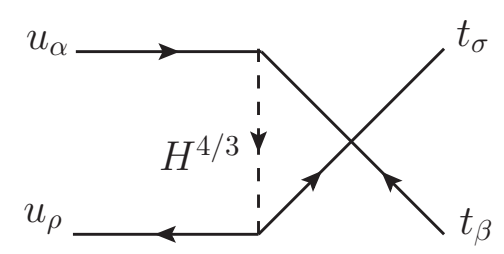

$(b)$

FIG. 1: Feynman diagrams for (a) $d \bar{d} \rightarrow t \bar{t}$ generated by models (1)-(5) and (b) $u \bar{u} \rightarrow t \bar{t}$ generated by models (6) and (7).

tion, we will not demonstrate the results of all models shown in Table I. Instead, we select the diquarks of $(3,1,-4 / 3)$ and $(6,1,4 / 3)$ as representative, where the $t \bar{t}$ pair is produced through a u-channel diagram with diquark exchange as depicted in Fig. 1, Accordingly, the interactions of color-triplet and sextet diquarks with quarks are respectively written by

$$
\begin{aligned}
& \mathcal{L}_{\mathbf{3}}=-f_{i j}^{\mathbf{3}} \bar{u}_{i \alpha} P_{L} u_{j \beta}^{c} \epsilon^{\alpha \beta \gamma} H_{\mathbf{3} \gamma}^{\dagger}+\text { h.c. }, \\
& \mathcal{L}_{\mathbf{6}}=-f_{i j}^{\mathbf{6}} \bar{u}_{i \alpha} P_{L} u_{j \beta}^{c} H_{\mathbf{6}}^{\alpha \beta}+\text { h.c. }
\end{aligned}
$$

where the indices $i$ and $j$ are the quark flavors, $f_{i j}^{\mathbf{3}}=-f_{j i}^{\mathbf{3}}, f_{i j}^{\mathbf{6}}=f_{j i}^{\mathbf{6}},(\alpha, \beta, \gamma)$ stand for the color indices, $\epsilon^{\alpha \beta \gamma}$ is antisymmetric tensor in color space and $P_{L(R)}=\left(1 \mp \gamma_{5}\right) / 2$ is the helicity projection. In terms of Eq. (2), the process $u \bar{u} \rightarrow t \bar{t}$ could proceed through the following interactions

$$
\mathcal{L}=2 f_{u t}^{\mathbf{3}} \bar{u}_{\alpha} P_{L} t_{\beta}^{c} \epsilon^{\alpha \beta \gamma} H_{\mathbf{3} \gamma}^{\dagger}+2 f_{u t}^{\mathbf{6}} \bar{u}_{\alpha} P_{L} t_{\beta}^{c} H_{\mathbf{6}}^{\alpha \beta}+\text { h.c. }
$$


Before presenting the amplitude of $u \bar{u} \rightarrow t \bar{t}$ mediated by gluon and the diquark $H$, let us first define a convenient notation for the following calculations. The momenta of the incoming up-quark and anti-up-quark, outgoing top and outgoing anti-top quarks are denoted by $p_{u}, p_{\bar{u}}, p_{t}$ and $p_{\bar{t}}$ respectively such that $p_{u}+p_{\bar{u}}=p_{t}+p_{\bar{t}}$. The momentum can be written as:

$$
\begin{aligned}
& p_{u, \bar{u}}=\frac{\sqrt{\hat{s}}}{2}(1,0,0, \pm 1) \\
& p_{t, \bar{t}}=\frac{\sqrt{\hat{s}}}{2}(1, \pm \beta \sin \theta, 0, \pm \beta \cos \theta)
\end{aligned}
$$

where $\beta^{2}=1-4 m_{t}^{2} / \hat{s}$ and $\theta$ is the scattering angle in the center-of-mass frame of the partons. Neglecting the up-quark masses of the incoming partons, the Mandelstam variables are defined as follow

$$
\begin{aligned}
& \hat{s}=\left(p_{u}+p_{\bar{u}}\right)^{2}=\left(p_{t}+p_{\bar{t}}\right)^{2}, \\
& \hat{t}=\left(p_{u}-p_{t}\right)^{2}=\left(p_{\bar{u}}-p_{\bar{t}}\right)^{2}=m_{t}^{2}-\frac{\hat{s}}{2}(1-\beta \cos \theta), \\
& \hat{u}=\left(p_{u}-p_{\bar{t}}\right)^{2}=\left(p_{\bar{u}}-p_{t}\right)^{2}=m_{t}^{2}-\frac{\hat{s}}{2}(1+\beta \cos \theta) .
\end{aligned}
$$

By combining both the gluon SM contribution and the diquark contribution at leading order, the amplitude can be written as

$$
\begin{aligned}
\mathcal{M}_{S M+H}(u \bar{u} \rightarrow t \bar{t})= & \frac{g_{s}^{2}}{\hat{s}}\left[\bar{v}_{u}\left(p_{\bar{u}}\right) \gamma^{\mu} T^{a} u_{u}\left(p_{u}\right)\right]\left[\bar{u}_{t}\left(p_{t}\right) \gamma_{\mu} T^{a} v_{t}\left(p_{\bar{t}}\right)\right] \\
- & \frac{\left|2 f_{u t}\right|^{2}}{2\left(\hat{u}-m_{H}^{2}\right)}\left[\bar{v}_{u}\left(p_{\bar{u}}\right) \gamma_{\mu} P_{R} u\left(p_{u}\right) \bar{u}_{t}\left(p_{t}\right) \gamma^{\mu} P_{R} v_{t}\left(p_{\bar{t}}\right)\right. \\
& \left.+\epsilon_{c} \bar{u}_{t \alpha}\left(p_{t}\right) \gamma^{\mu} P_{R} v_{t}^{\beta}\left(p_{\bar{t}}\right) \bar{v}_{u \beta}\left(p_{\bar{u}}\right) \gamma_{\mu} P_{R} u_{u}^{\alpha}\left(p_{u}\right)\right]
\end{aligned}
$$

where $\epsilon_{c}=-(+)$ denotes the color triplet (sextet), $T^{a}$ are the generators of $S U(3)_{c}$ group, $g_{s}$ is the gauge coupling constant. In addition, we have adopted the Fierz transformation $\bar{q}_{1} P_{L} q_{2} \bar{q}_{3} P_{R} q_{4}=(\xi / 2) \bar{q}_{1} \gamma^{\mu} P_{R} q_{4} \bar{q}_{3} \gamma_{\mu} P_{L} q_{2}$ and $\bar{q}_{1}^{c} \gamma_{\mu} P_{L} q_{2}^{c}=\xi \bar{q}_{2} \gamma_{\mu} P_{R} q_{1}$ in which $\left\{q_{i}\right\}$ are the spinors and $\xi=+(-)$ when $\left\{q_{i}\right\}$ are regarded as c-numbers (field operators). Then, the squared amplitude takes the following form

$$
\begin{aligned}
\sum \overline{\left|\mathcal{M}_{S M+H}\right|^{2}} & =\frac{4 \pi^{2} \alpha_{s}^{2}}{N_{c}^{2}}\left(N_{c}^{2}-1\right)\left(1+\beta^{2} \cos ^{2} \theta+\frac{4 m_{t}^{2}}{\hat{s}}\right) \\
& -\frac{\pi \alpha_{s}}{N_{c}^{2}} \epsilon_{c}\left(\frac{N_{c}^{2}-1}{2}\right) \frac{f_{u t}^{\prime 2} \hat{s}}{\hat{u}-m_{H}^{2}}\left((1+\beta \cos \theta)^{2}+\frac{4 m_{t}^{2}}{\hat{s}}\right) \\
& +\frac{1}{8 N_{c}^{2}} N_{c}\left(N_{c}+\epsilon_{c}\right) \frac{f_{u t}^{\prime 4} \hat{s}^{2}}{\left(\hat{u}-m_{H}^{2}\right)^{2}}(1+\beta \cos \theta)^{2}
\end{aligned}
$$


where we have already summed over final state color and averaged over the initial spin and color, $f_{u t}^{\prime}=2 f_{u t}$ and $N_{c}=3$. We note that since the propagator in the $u$-channel diagram depends on the scattering angle $\theta$, the FBA may arise not only from $\cos \theta$ term in Eq. (77), but also from the constant term and the $\cos ^{2} \theta$ term. The partonic differential cross section for the subprocess $u \bar{u} \rightarrow t \bar{t}$ in the parton rest frame is given by

$$
\frac{d \hat{\sigma}}{d \cos \theta}=\frac{\beta}{32 \pi \hat{s}} \sum \overline{\left|\mathcal{M}_{S M+H}\right|^{2}}
$$

To obtain the hadronic cross section $\sigma$, the partonic one is then convoluted with the parton distribution functions. In our study we have used PDF distribution CTEQ6 [18] at the leading order and set the renormalization and factorization scales to $\mu_{F}=\mu_{R}=m_{t}$. Accordingly, the FBA in top-quark pair production is defined by

$$
A_{F B}^{t}=\frac{\sigma(\eta \geq 0)-\sigma(\eta \leq 0)}{\sigma(\eta \geq 0)+\sigma(\eta \leq 0)}
$$

where $\eta$ denotes the rapidity of top quarks and is defined by $\eta=2 \tanh ^{-1}(\beta \cos \theta)$.

Before presenting our numerical analysis, it is worth discussing the strict constraints by other low energy processes such as $D^{0}-\bar{D}^{0}$ mixing. It has been studied in Ref. [19] that the diquark of $(6,1,4 / 3)$ representation could contribute to $D^{0}-\bar{D}^{0}$ mixing by a tree-diagram while the diquark of $(3,1,-4 / 3)$ representation would contribute through the box-diagram. Nevertheless, since the related parameter for the former is $f_{u c}$ while the latter is $f_{u t} f_{c t}^{*}$, by setting $f_{c t}$ to be small enough, $f_{u t}$ could escape the direct constraint from $D^{0}-\bar{D}^{0}$ mixing. In other words, $f_{u t}$ could be taken as a free parameter and could be of the order of unity in our study. The only involved free parameters are the coupling $f_{u t}$ and the diquark mass $m_{H}$, which could be constrained with the use of the experimental measurement of $t \bar{t}$ cross section $\sigma(p \bar{p} \rightarrow t \bar{t})$ from [23]

$$
\sigma(p \bar{p} \rightarrow t \bar{t})^{\exp }=7.5 \pm 0.31(\text { stat }) \pm 0.34(\text { syst }) \pm 0.15(\text { th }) \quad \mathrm{pb}
$$

where the SM prediction is $\sigma(p \bar{p} \rightarrow t \bar{t})^{\mathrm{SM}}=6.73_{-0.79}^{+0.71} \mathrm{pb}$ [22]. In our numerical estimation, we use $m_{t}=172.5 \mathrm{GeV}$ with $\alpha_{s}=0.1095$ we take as input the experimental value given by Eq. (10) with $1 \sigma$ errors. In addition, for matching the cross section of $p \bar{p} \rightarrow t \bar{t}$ up to the next-to-leading order in the SM [22], a QCD K-factor of 1.3 has been multiplied everywhere in our calculations.

With the above inputs, we are now ready to present our numerical analysis for the contributions of color-triplet and -sextet diquarks separately. In terms of Eqs. (77) and 
(8) and with $\epsilon_{c}=-1$, the inclusive cross section for $t \bar{t}$ production mediated by gluons and triplet diquark is displayed in Fig. 2(a), where the solid, dashed and dash-dotted lines denote the influence of triplet diquark with $m_{H}=300,500$ and $800 \mathrm{GeV}$, respectively. The band shown in the plot is the experimental measurement of $t \bar{t}$ cross section with $1 \sigma$ errors. Clearly, $\sigma(p \bar{p} \rightarrow t \bar{t})$ is sensitive to the diquark contribution. Moreover, by Eq. (91), we present the FBA in top-quark pair production in Fig. 2(b). When we contrast Fig. 2(a) with Fig. 2(b), it is clear that with the same values of parameters, triplet diquark can fit both the FBA of $(10-30) \%$ as well as $t \bar{t}$ cross section $\sigma(p \bar{p} \rightarrow t \bar{t})^{\exp }$ simultaneously. As it can be seen from Fig. 2, this could happen when $f_{u t}^{\prime}$ is located in the range $(1.67,1.83),(2.22,2.43)$ and $(3.04,3.39)$ for $m_{H}=300,500$ and $800 \mathrm{GeV}$, respectively.
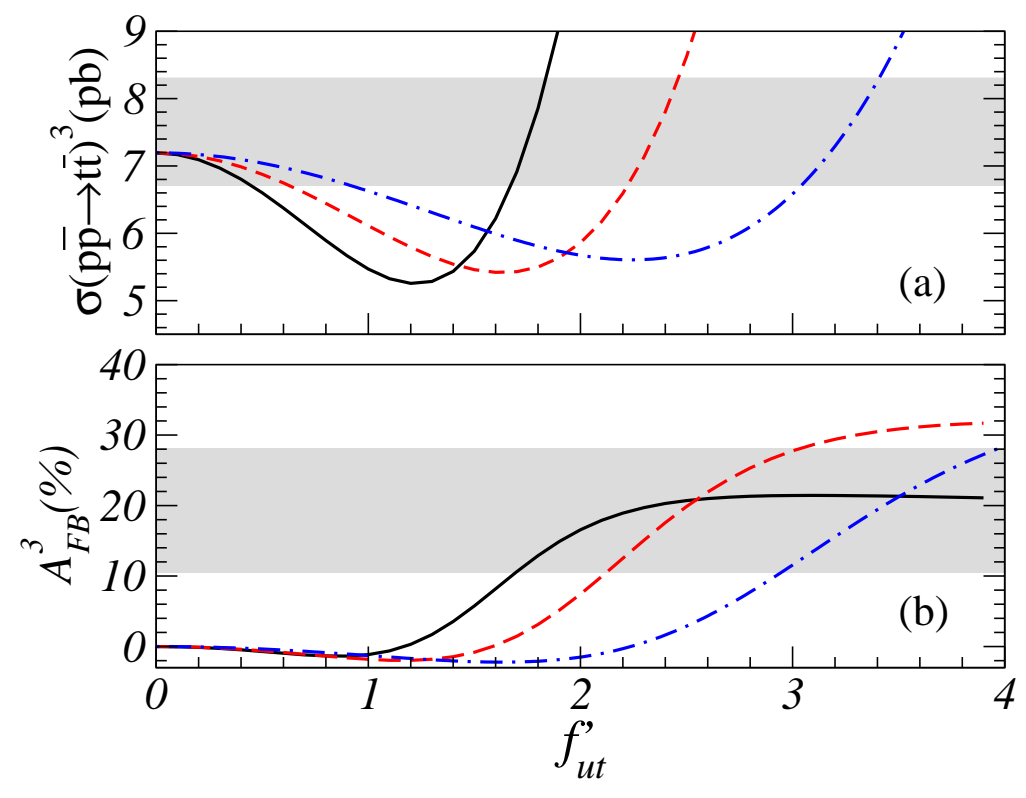

FIG. 2: (a) Cross section for inclusive top-quark pair production and (b) FBA of top-quark in triplet diquark model, where the solid, dashed and dash-doted lines denote $m_{H}=300,500$ and $800 \mathrm{GeV}$, respectively. The bands in the plots denote the experimental data with $1 \sigma$ errors.

Similarly, with $\epsilon_{c}=+1$, the $t \bar{t}$ cross section $\sigma(p \bar{p} \rightarrow t \bar{t})$ mediated by sextet diquark as a function of $f_{u t}^{\prime}$ is illustrated in Fig. 3(a), in which the solid, dashed and dash-dotted lines represent the contributions from $m_{H}=300,500$ and $800 \mathrm{GeV}$, respectively. In Fig. 2(a) and Fig. 3(a), we can see that the behavior of triplet and sextet contribution in $t \bar{t}$ production is quite different. In some region of $f_{u t}^{\prime}$, the interference between triplet diquark and SM-gluon contribution is destructive. In contract, the interference between the sextet diquark and 
SM-gluon contribution is all the time constructive. This different behavior between triplet and sextet is mainly due to the sign of the 2nd term in Eq. (7) for which the triplet diquark is negative while the sextet diquark is positive. Furthermore, we show the associated $A_{F B}^{t}$ defined by Eq. (9) in Fig. 3(b). From Figs. 3(a) and 3(b), one can easily see that the sextet diquark could not fit the current data of $A_{F B}^{t}$ and $\sigma(p \bar{p} \rightarrow t \bar{t})^{\exp }$ simultaneously.
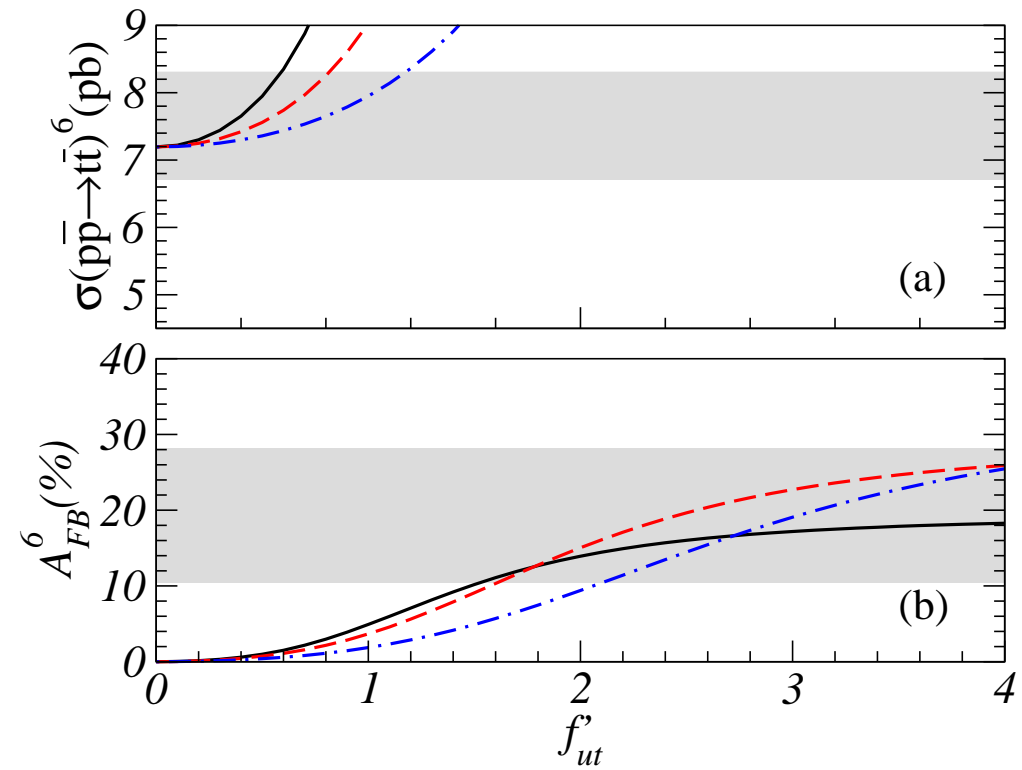

FIG. 3: Legend is the same as Fig. 2 but for sextet diquark model.

According to previous analysis, we have shown that color-triplet diquark plays an important role on the top-quark FBA. To be more clear, we have shown how $\sigma(p \bar{p} \rightarrow t \bar{t})$ and $A_{F B}^{t}$ are affected by the free parameters of the diquark models. For this purpose, we perform a systematic scan over the parameters $f_{u t}^{\prime}$ and $m_{H}$ of the triplet diquark. In Fig. 4(a), we show the allowed values of $f_{u t}^{\prime}$ and $m_{H}$ that satisfy the measurement of $\sigma(p \bar{p} \rightarrow t \bar{t})$ within $1 \sigma$ errors. Furthermore, with those allowed values, we make a two-dimensional contour as a function of $f_{u t}^{\prime}$ and $m_{H}$ in Fig. 4(b), where the labels in the plot denote the corresponding values of $A_{F B}^{t}$. From the contour plot, not only can one easily see the influence of triplet diquark on the FBA of top-quark, but also one can understand the correlation between $f_{u t}^{\prime}$ and $m_{H}$.

In the present analysis, we have shown the effects of colored scalars on the FBA in top-quark production. If the diquarks particles with a mass of the order $O(100 \mathrm{GeV})$ exit in the universe, they could be produced at hadron colliders such as Tevatron and LHC. Those diquarks can be produced through the annihilation of a pair of up quarks $u u \rightarrow H_{6}$ 

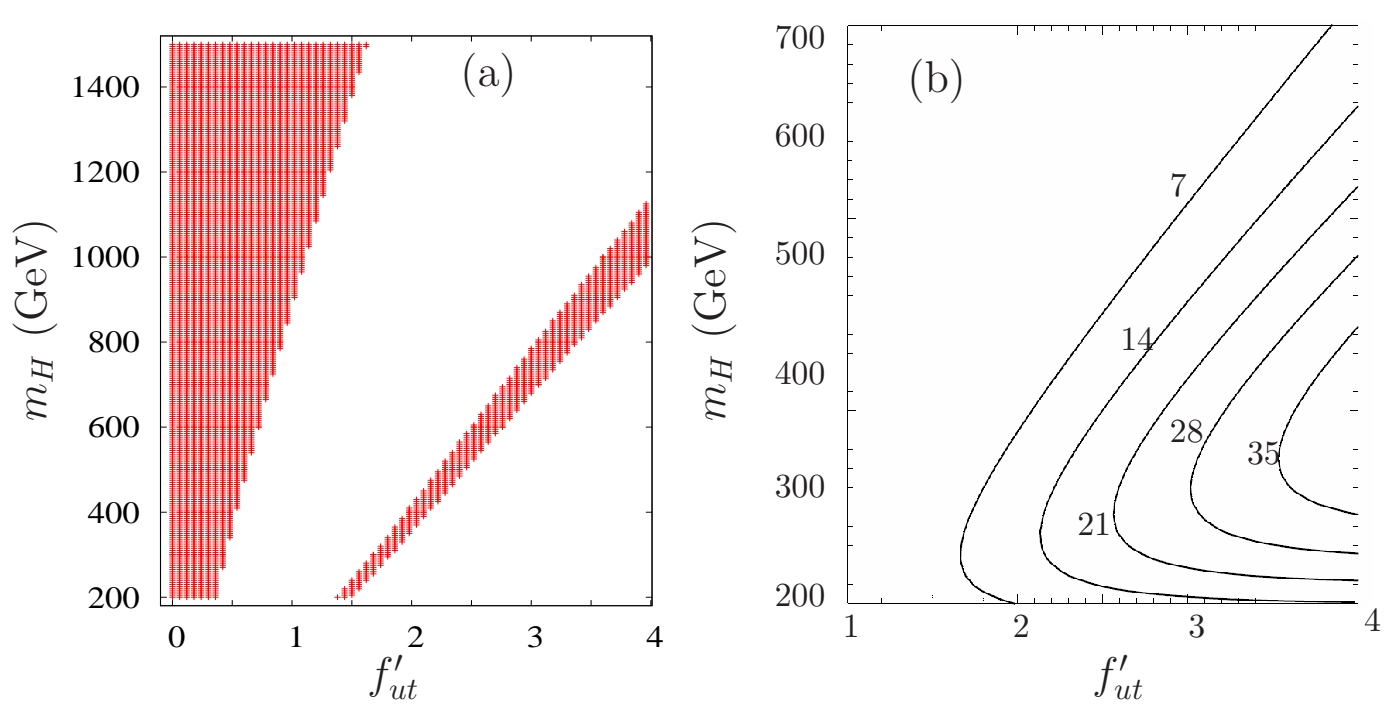

FIG. 4: (a) The allowed range of $f_{u t}^{\prime}$ and $m_{H}$ by fitting the measurement of $\sigma(p \bar{p} \rightarrow t \bar{t})^{\exp }$ with $1 \sigma$ errors. (b) Contour for FBA as a function of $f_{u t}^{\prime}$ and $m_{H}$, where the results in (a) have been applied.

[21, 24] and in association with top-quark through $u g \rightarrow \bar{t} H_{\mathbf{3}, \mathbf{6}}$ [10]. Scalar diquark can also be produced in pair, such production process proceeds through the QCD couplings of the diquark. The general cross section of scalar diquark pair production is given in the first reference of [24] at the tree-level. Here, we plot in Fig. 5, scalar triplet pair production at the LHC with different energies. The factorization scale has been set to $\mu_{F}=m_{H_{3}}$. As we can see, the total production cross section of the triplet scalar can reach $17 \mathrm{fb}$, $78 \mathrm{fb}$ and 2.6pb for 7, 10 and $14 \mathrm{TeV}$, respectively for Diquark mass of $400 \mathrm{GeV}$. Note that for LHC with $14 \mathrm{TeV}$ with low luminosity of $1 \mathrm{fb}^{-1}$ would lead to 2600 raw events which could be enough to extract a signal.

If kinematically allowed, the scalar diquark would then decay into $t t \overline{t t}$ in the case of pair production which would lead to same-sign dileptons signal. Single production of scalar diquark can lead to double top $t t$ and single top $t u$, tc plus jet production depending on its mass, coupling and the representation of colored scalar. Note that some decay channel of the diquark such as $t$, which could lead to same-sign dileptons plus multijets if both top-quarks decay semileptonically, does not exist in SM and would be, in principle, easily distinguished from the SM background. At the Tevatron, the production rate of diquark is rather small, whereas the production rate of diquark is rather large at the LHC [24]. Hence, the LHC provides a good environment to discover the diquarks and study their properties. 
In summary, an unexpected large FBA of top-quark has been observed by CDF and D $\varnothing$

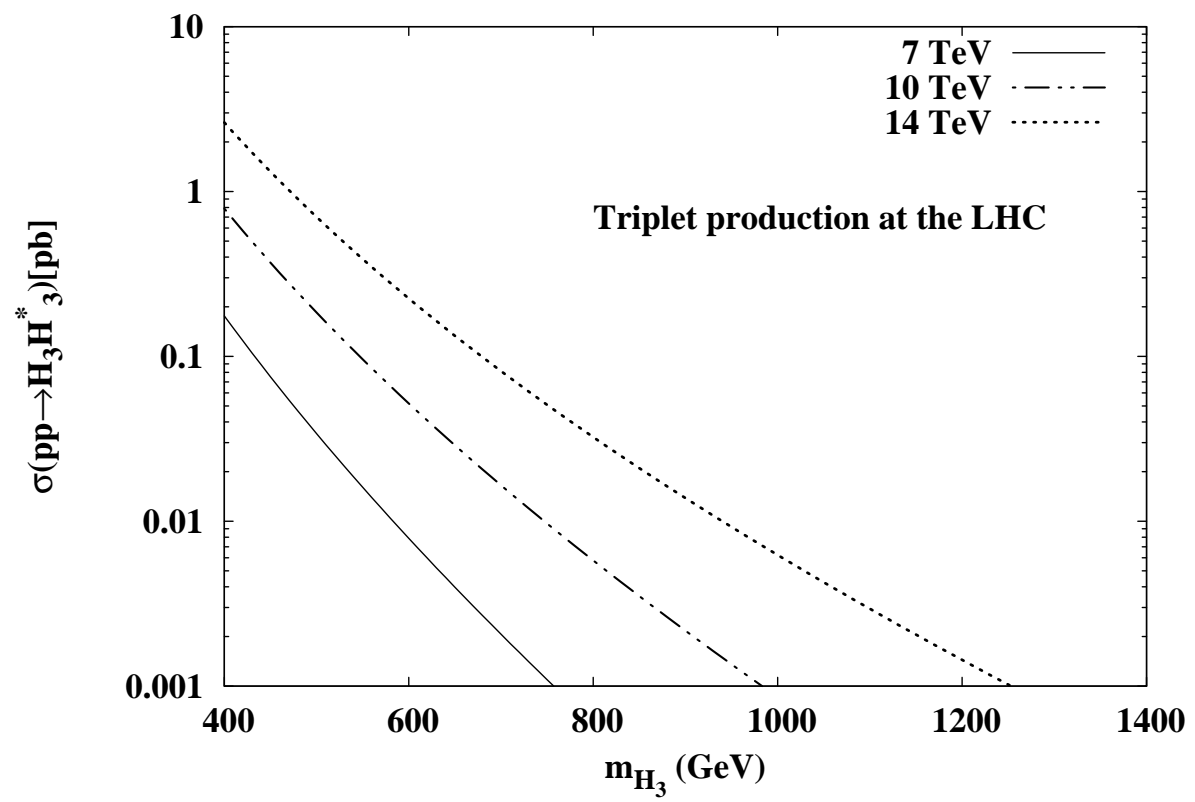

FIG. 5: Hadronic production cross section of triplet $H_{3}$ as a function of triplet mass $m_{H_{3}}$ at the LHC for $\sqrt{s}=7,10$ and $14 \mathrm{TeV}$.

Collaborations [1-3]. To account for this anomaly, we study the contribution of diquarks to the FBA in top-quark production. We find that the parameters of diquark models involved in $u \bar{u} \rightarrow t \bar{t}$ can escape the strict limit imposed by $D^{0}-\bar{D}^{0}$ mixing, therefore the measured cross section of top-pair production at the Tevatron is the main constraint. We show that with the current data of $\sigma(p \bar{p} \rightarrow t \bar{t})$ within $1 \sigma$ errors, only color triplet diquark can give a large and consistent FBA with the updated measurement of CDF [3], while the color-sextet representation can not fit simultaneously the $A_{F B}^{t}$ and $\sigma(p \bar{p} \rightarrow t \bar{t})^{\exp }$.

Note added: While we were finishing the present work, we received a paper [11] dealing with similar subject. Our result agrees with [11] in the case of color-triplet diquark. However, our results for triplet and sextet diquarks are consistent with the paper [25].

\section{Acknowledgments}

We thank Dr. J. Shu and Dr. J. F. Kamenik for their useful discussions on the color factor and the sign convention in the interference between SM and diquark contributions. 
A.A thanks LPTA University Montpellier-II for hospitality extended to him during his visit, project AI $N^{0}$ MA-186-08, where part of this work has been done. R.B is supported by National Cheng Kung University Grant No. HUA 97-03-02-063. R.B acknowledges the KEK theory exchange program for physicists in Taiwan and the very kind hospitality at KEK. C.C.H is supported by the National Science Council of R.O.C under Grant \#s: NSC97-2112-M-006-001-MY3.

[1] V. M. Abazov et al. [D0 Collaboration], Phys. Rev. Lett. 100, 142002 (2008) arXiv:0712.0851 [hep-ex]].

[2] T. Aaltonen et al. [CDF Collaboration], Phys. Rev. Lett. 101, 202001 (2008) arXiv:0806.2472 [hep-ex]].

[3] CDF note at http://www-cdf.fnal.gov/physics/new/top/2009/tprop/Afb/.

[4] O. Antunano, J. H. Kuhn and G. Rodrigo, Phys. Rev. D 77, 014003 (2008) arXiv:0709.1652 [hep-ph]].

[5] A. Djouadi, G. Moreau, F. Richard and R. K. Singh, arXiv:0906.0604 [hep-ph].

[6] P. Ferrario and G. Rodrigo, Phys. Rev. D 80, 051701 (2009) arXiv:0906.5541 [hep-ph]].

[7] S. Jung, H. Murayama, A. Pierce and J. D. Wells, arXiv:0907.4112 [hep-ph].

[8] K. Cheung, W. Y. Keung and T. C. Yuan, arXiv:0908.2589 [hep-ph].

[9] P. H. Frampton, J. Shu and K. Wang, arXiv:0911.2955 [hep-ph].

[10] J. Shu, T. M. P. Tait and K. Wang, arXiv:0911.3237 [hep-ph].

[11] I. Dorsner, S. Fajfer, J. F. Kamenik and N. Kosnik, arXiv:0912.0972 [hep-ph].

[12] S. M. Barr, Phys. Rev. D 34, 1567 (1986).

[13] S. M. Barr and E. M. Freire, Phys. Rev. D 41, 2129 (1990).

[14] R. Barbier et al., Phys. Rept. 420, 1 (2005) arXiv:hep-ph/0406039.

[15] Z. Chacko and R. N. Mohapatra, Phys. Rev. D 59, 055004 (1999) arXiv:hep-ph/9802388.

[16] A. V. Manohar and M. B. Wise, Phys. Rev. D 74, 035009 (2006) arXiv:hep-ph/0606172.

[17] J. M. Arnold, M. Pospelov, M. Trott and M. B. Wise, arXiv:0911.2225 [hep-ph].

[18] S. Kretzer, H. L. Lai, F. I. Olness and W. K. Tung, Phys. Rev. D 69, 114005 (2004).

[19] C. H. Chen, Phys. Lett. B680, 113 (2009) arXiv:0902.2620 [hep-ph]].

[20] A. Abulencia et al. [CDF Collaboration], Phys. Rev. Lett. 98, 221803 (2007) 
arXiv:hep-ex/0702051.

[21] R. N. Mohapatra, N. Okada and H. B. Yu, Phys. Rev. D 77, 011701 (2008) arXiv:0709.1486 [hep-ph]].

[22] M. Cacciari et al., JHEP 0404, 068 (2004) arXiv:hep-ph/0303085; M. Cacciari et al., JHEP 0809, 127 (2008) arXiv:0804.2800 [hep-ph]]; N. Kidonakis and R. Vogt, Phys. Rev. D 78, 074005 (2008) arXiv:0805.3844 [hep-ph]]; S. Moch and P. Uwer, Phys. Rev. D 78, 034003 (2008) arXiv:0804.1476 [hep-ph]].

[23] Combination of CDF top pair production cross section could be found at http://www-cdf.fnal.gov/physics/new/top/2009/xsection/old_ttbar_combined_46invfb/

[24] C. R. Chen et al, Phys. Rev. D 79, 054002 (2009) arXiv:0811.2105 [hep-ph]]; T. Han, I. Lewis and T. McElmurry, arXiv:0909.2666 [hep-ph].

[25] D. W. Jung, P. Ko, J. S. Lee and S. h. Nam, arXiv:0912.1105 [hep-ph]. 